\title{
The matrix version for the multivariable Humbert polynomials
}

\author{
Rabia Aktaş, Bayram Çekim, and Recep Şahin
}




\title{
THE MATRIX VERSION FOR THE MULTIVARIABLE HUMBERT POLYNOMIALS
}

\author{
RABİA AKTAŞ, BAYRAM ÇEKİM, AND RECEP ŞAHİN
}

Received 4 May, 2011

\begin{abstract}
In this paper, the matrix extension of the multivariable Humbert polynomials is introduced. Various families of linear, multilinear and multilateral generating matrix functions of these matrix polynomials are presented. Miscellaneous applications are also discussed.
\end{abstract}

2000 Mathematics Subject Classification: 33C25; 15A60

Keywords: Humbert polynomials, Chan-Chyan-Srivastava polynomials, Lagrange-Hermite polynomials, generating matrix function, matrix functional calculus

\section{INTRODUCTION}

It is well-known that special matrix functions appear in the study of many areas. Generalization of the property of orthogonality [11,12], Rodrigues formula [6, 10], a second-order Sturm-Liouville differential equation [10], a three-term matrix recurrence [6,7], relation between different orthogonal matrix polynomials [21] are theoretical examples. Statistics, group representation theory [17], scattering theory [15], differential equations [18, 19], Fourier series expansions [9], interpolation and quadrature [22, 23], splines [8], and medical imaging [5] are areas of application of orthogonal matrix polynomials.

Throughout this paper, for a matrix $A \in \mathbb{C}^{N \times N}$, its spectrum is denoted by $\sigma(A)$. The two-norm of $A$, which will be denoted by $\|A\|$, is defined by

$$
\|A\|=\sup _{x \neq 0} \frac{\|A x\|_{2}}{\|x\|_{2}}
$$

where, for a vector $y \in \mathbb{C}^{N},\|y\|_{2}=\left(y^{T} y\right)^{1 / 2}$ is the Euclidean norm of $y . I$ and $\theta$ will denote the identity matrix and the null matrix in $\mathbb{C}^{N \times N}$, respectively. We say that a matrix $A$ in $\mathbb{C}^{N \times N}$ is a positive stable if $\Re(\lambda)>0$ for all $\lambda \in \sigma(A)$, where $\sigma(A)$ is the set of the eigenvalues of $A$. If $A_{0}, A_{1}, \ldots, A_{n}$ are elements of $\mathbb{C}^{N \times N}$ and $A_{n} \neq \theta$, then we call

$$
P(x)=A_{n} x^{n}+A_{n-1} x^{n-1}+\ldots+A_{1} x+A_{0}
$$


a matrix polynomial of degree $n$ in $x$. From [20], one can see

$$
(P)_{n}=P(P+I)(P+2 I) \ldots(P+(n-1) I) ; n \geq 1 ;(P)_{0}=I .
$$

For any matrix $A$ in $\mathbb{C}^{N \times N}$, the authors exploited the following relation due to [20]

$$
(1-x)^{-A}=\sum_{n=0}^{\infty} \frac{(A)_{n}}{n !} x^{n},|x|<1 .
$$

Gould [16] presented a systematic study of an interesting generalization of the Humbert and the Gegenbauer polynomials and several other polynomial systems, that is called generalized Humbert polynomials and defined by

$$
\left(C-m x t+y t^{m}\right)^{p}=\sum_{n=0}^{\infty} P_{n}(m, x, y, p, C) t^{n}
$$

where $m$ is a positive integer and the other parameters are unrestricted (see also [26, p. 77, 86] ).

Aktas et al. [1] present a systematic investigation of a multivariable extension of the Humbert polynomials generated by

$$
\begin{gathered}
\prod_{i=1}^{r}\left\{\left(C_{i}-m_{i} x_{i} t+y_{i} t^{m_{i}}\right)^{-\alpha_{i}}\right\}=\sum_{n=0}^{\infty} P_{n}^{\left(\alpha_{1}, \ldots, \alpha_{r}\right)}(\mathbf{m}, \mathbf{x}, \mathbf{y}, \mathbf{C}) t^{n} \\
\left(\left|m_{i} x_{i} t-y_{i} t^{m_{i}}\right|<\left|C_{i}\right| ; i=1,2, \ldots, r\right)
\end{gathered}
$$

where $\mathbf{x}=\left(x_{1}, \ldots, x_{r}\right), \mathbf{y}=\left(y_{1}, \ldots, y_{r}\right), \mathbf{C}=\left(C_{1}, \ldots, C_{r}\right), \mathbf{m}=\left(m_{1}, \ldots, m_{r}\right), m_{i}=$ $1,2, \ldots(i=1,2, \ldots, r)$ and the other parameters are unrestricted.

The main objective of this paper is to construct a matrix version of the multivariable Humbert polynomials given by (1.4) and the derivation of various families of multilinear and mixed multilateral generating matrix functions for these matrix

polynomials. We present some special cases of our results and also obtain several recurrence relations for these matrix polynomials.

\section{MATRIX EXTENSION OF THE MULTIVARIABLE Humbert POLYNOMIALS}

The main object of this section is to present a systematic investigation of the matrix extension of the multivariable Humbert polynomials generated by

$$
\begin{gathered}
\prod_{i=1}^{r}\left\{\left(C_{i}-m_{i} x_{i} t+y_{i} t^{m_{i}}\right)^{-A_{i}}\right\}=\sum_{n=0}^{\infty} P_{n}^{\left(A_{1}, \ldots, A_{r}\right)}(\mathbf{m}, \mathbf{x}, \mathbf{y}, \mathbf{C}) t^{n} \\
\left(\left|m_{i} x_{i} t-y_{i} t^{m_{i}}\right|<\left|C_{i}\right| ; i=1,2, \ldots, r\right)
\end{gathered}
$$

where $A_{i} \in \mathbb{C}^{N \times N}, \mathbf{x}=\left(x_{1}, \ldots, x_{r}\right), \mathbf{y}=\left(y_{1}, \ldots, y_{r}\right), \mathbf{C}=\left(C_{1}, \ldots, C_{r}\right)$, $\mathbf{m}=\left(m_{1}, \ldots, m_{r}\right), m_{i}=1,2, \ldots(i=1,2, \ldots, r)$ and the other parameters are unrestricted. 
(2.1) yields the following explicit representation:

$$
\begin{aligned}
& P_{n}^{\left(A_{1}, \ldots, A_{r}\right)}(\mathbf{m}, \mathbf{x}, \mathbf{y}, \mathbf{C}) \\
= & \sum_{m_{1} k_{1}+\ldots+m_{r} k_{r}+n_{1}+\ldots+n_{r}=n} \\
& \frac{\left(A_{1}\right)_{n_{1}+k_{1}} C_{1}^{-A_{1}-\left(n_{1}+k_{1}\right) I} \ldots\left(A_{r}\right)_{n_{r}+k_{r}} C_{r}^{-A_{r}-\left(n_{r}+k_{r}\right) I}}{n_{1} ! \ldots n_{r} ! k_{1} ! \ldots k_{r} !} \\
& \times m_{1}^{n_{1}} \ldots m_{r}^{n_{r}}(-1)^{k_{1}+\ldots+k_{r}} x_{1}^{n_{1}} \ldots x_{r}^{n_{r}} y_{1}^{k_{1}} \ldots y_{r}^{k_{r}} \\
= & \left.\sum_{1}^{r} \prod_{k_{1}+\ldots+m_{r} k_{r}+n_{1}+\ldots+n_{r}=n} m_{p=1}^{n_{p}}(-1)^{k_{p}} x_{p}^{n_{p}} y_{p}^{k_{p}}\right\} \\
& \left\{\frac{\left(A_{p}\right)_{n_{p}+k_{p}} C_{p}^{-A_{p}-\left(n_{p}+k_{p}\right) I}}{n_{p} ! k_{p} !}\right.
\end{aligned}
$$

where, as usual, $(A)_{n}$ denotes the Pochhammer symbol given by (1.1).

We notice that the case $r=1$ in (2.1) reduces to the matrix version of the generalized Humbert polynomials introduced by Gould [16]. In this case, it is generated by

$$
\left(C-m x t+y t^{m}\right)^{-A}=\sum_{n=0}^{\infty} P_{n}^{(A)}(m, x, y, C) t^{n}
$$

where $\left|m x t-y t^{m}\right|<|C|, A \in \mathbb{C}^{N \times N}, m$ is a positive integer and the other parameters are unrestricted. For the special cases of (2.3), including Gegenbauer matrix polynomials, we refer [19].

It is clear that the case

$$
C_{i}=1, m_{i}=1, y_{i}=0, i=1,2, \ldots, r
$$

of the polynomials of (2.1) reduces to matrix version of the Chan-Chyan-Srivastava multivariable polynomials, which is generated by [14]

$$
\begin{gathered}
\prod_{i=1}^{r}\left\{\left(1-x_{i} t\right)^{-A_{i}}\right\}=\sum_{n=0}^{\infty} \mathrm{g}_{n}^{\left(A_{1}, \ldots, A_{r}\right)}\left(x_{1}, \ldots, x_{r}\right) t^{n} \\
\left(A_{i} \in \mathbb{C}^{N \times N}(i=1,2, \ldots, r) ;|t|<\min \left\{\left|x_{1}\right|^{-1}, \ldots,\left|x_{r}\right|^{-1}\right\}\right) .
\end{gathered}
$$

Since $A_{i}=\alpha_{i} \in \mathbb{C}$ for $N=1$ in (2.4), we obtained the generating function of the Chan-Chyan-Srivastava multivariable polynomials [3].

On the other hand, if we choose $C_{i}=1, m_{i}=i, x_{i}=0, y_{i}=-x_{i}, \quad i=$ $1,2, \ldots, r$ in $(2.1)$, we get a matrix version of the multivariable Lagrange-Hermite 
polynomials, which is generated by [14]

$$
\begin{gathered}
\prod_{i=1}^{r}\left\{\left(1-x_{i} t^{i}\right)^{-A_{i}}\right\}=\sum_{n=0}^{\infty} h_{n}^{\left(A_{1}, \ldots, A_{r}\right)}\left(x_{1}, \ldots, x_{r}\right) t^{n} \\
\left(A_{i} \in \mathbb{C}^{N \times N}(i=1,2, \ldots, r) ;|t|<\min \left\{\left|x_{1}\right|^{-1},\left|x_{2}\right|^{-1 / 2}, \ldots,\left|x_{r}\right|^{-1 / r}\right\}\right) .
\end{gathered}
$$

Since $A_{i}=\alpha_{i} \in \mathbb{C}$ for $N=1$ in (2.5), we have the multivariable Lagrange-Hermite polynomials presented by Altın and Erkuş [2]. Furthermore, we should remark that the case $r=2$ of the polynomials corresponds to the familiar (two-variable) Lagrange-Hermite polynomials considered by Dattoli et al. [4].

Moreover, the special case

$$
C_{i}=1, x_{i}=0, y_{i}=-x_{i}, \quad i=1,2, \ldots, r
$$

gives the matrix version of the Erkus-Srivastava multivariable polynomials generated by [14]

$$
\begin{gathered}
\prod_{i=1}^{r}\left\{\left(1-x_{i} t^{m_{i}}\right)^{-A_{i}}\right\}=\sum_{n=0}^{\infty} u_{n}^{\left(A_{1}, \ldots, A_{r}\right)}\left(x_{1}, \ldots, x_{r}\right) t^{n}, \\
A_{i} \in \mathbb{C}^{N \times N}(i=1,2, \ldots, r), \\
|t|<\min \left\{\left|x_{1}\right|^{-1 / m_{1}},\left|x_{2}\right|^{-1 / m_{2}}, \ldots,\left|x_{r}\right|^{-1 / m_{r}}\right\} .
\end{gathered}
$$

Since $A_{i}=\alpha_{i} \in \mathbb{C}$ for $N=1$ in (2.6), we have the Erkus-Srivastava multivariable polynomials generated by [13].

\section{AN APPLICATION OF SRIVASTAVA'S THEOREM ON MIXED GENERATING FUNCTIONS}

Srivastava [25] (see also the subsequent treatise on the subject by Srivastava and Manocha [26, p. 378, Theorem 12]) obtained a family of mixed generating functions for certain general multivariable and multiparameter sequences of functions. Our generating function (2.1) fits easily into the general setting of Srivastava's theorem. Thus, by applying this general result to the generating function (2.1), we obtain the 
following family of mixed generating functions for matrix version of the multivariable Humbert polynomials given by (2.1)

$$
\begin{aligned}
& \sum_{n=0}^{\infty} P_{n}^{\left(A_{1}+\lambda_{1} n I, \ldots, A_{r}+\lambda_{r} n I\right)}(\mathbf{m}, \mathbf{x}, \mathbf{y}, \mathbf{C}) t^{n}= \\
& \frac{\prod_{i=1}^{r}\left\{\left(C_{i}-m_{i} x_{i} \xi+y_{i} \xi^{m_{i}}\right)^{-A_{i}}\right\}}{1-\xi\left\{\sum_{i=1}^{r} \lambda_{i} m_{i}\left(C_{i}+y_{i} \xi^{m_{i}}\right)^{-1}\left[-y_{i} \xi^{m_{i}-1}+x_{i} \frac{\left(C_{i}-m_{i} y_{i} \xi^{m_{i}}+y_{i} \xi^{m_{i}}\right)}{\left(C_{i}-m_{i} x_{i} \xi+y_{i} \xi^{m_{i}}\right)}\right]\right\}} \\
& \left(\xi=\xi(t):=t \prod_{i=1}^{r}\left\{\left(C_{i}-m_{i} x_{i} \xi+y_{i} \xi^{m_{i}}\right)^{-\lambda_{i}}\right\} ; \lambda_{i} \in \mathbb{C},\right. \\
& \left.C_{i} \neq 0 \text { and } A_{i} \in \mathbb{C}^{N \times N}(i=1, \ldots, r)\right)
\end{aligned}
$$

where all of the matrices commute with each other. In a special case, it is easily seen that (3.1) would at once reduce to the generating function (2.1) when $\lambda_{i}=$ $0(i=1, \ldots, r)$. For the special case of $N=1,(3.1)$ gives mixed generating function for the multivariable Humbert polynomials given by [1]. Furthermore, the special case of $N=1$ and $r=1$ of (3.1) reduces to the mixed generating function for the generalized Humbert polynomials in [24].

\section{BILINEAR AND BILATERAL GENERATING MATRIX FUNCTIONS}

In this section, we derive several families of bilinear and bilateral generating matrix functions for matrix version of the multivariable Humbert polynomials which are generated by (2.1) and given explicitly by (2.2).

We begin by stating the following theorem.

Theorem 1. Corresponding to an identically non-vanishing function $\Omega_{\mu}(\mathbf{z})$ of $s$ complex variables $z_{1}, \ldots, z_{s}(s \in \mathbb{N})$ and of complex order $\mu$, let

$$
\Lambda_{\mu, \nu}(\mathbf{z} ; w):=\sum_{k=0}^{\infty} a_{k} \Omega_{\mu+v k}(\mathbf{z}) w^{k}
$$

where $\left(a_{k} \neq 0, \mu, v \in \mathbb{C}\right) ; \mathbf{z}=\left(z_{1}, \ldots, z_{s}\right)$ and

$$
\Theta_{n, p, \mu, v}(\mathbf{x}, \mathbf{y} ; \mathbf{z} ; \zeta):=\sum_{k=0}^{[n / p]} a_{k} P_{n-p k}^{\left(A_{1}, \ldots, A_{r}\right)}(\mathbf{m}, \mathbf{x}, \mathbf{y}, \mathbf{C}) \Omega_{\mu+v k}(\mathbf{z}) \zeta^{k}
$$


where $n, p \in \mathbb{N} ; A_{i} \in \mathbb{C}^{N \times N} ; \mathbf{x}=\left(x_{1}, \ldots, x_{r}\right) ; \mathbf{y}=\left(y_{1}, \ldots, y_{r}\right) ; \mathbf{C}=\left(C_{1}, \ldots, C_{r}\right)$; $\mathbf{m}=\left(m_{1}, \ldots, m_{r}\right), m_{i}=1,2, \ldots(i=1,2, \ldots, r)$. Then we have

$$
\sum_{n=0}^{\infty} \Theta_{n, p, \mu, \nu}\left(\mathbf{x}, \mathbf{y} ; \mathbf{z} ; \frac{\eta}{t^{p}}\right) t^{n}=\prod_{i=1}^{r}\left\{\left(C_{i}-m_{i} x_{i} t+y_{i} t^{m_{i}}\right)^{-A_{i}}\right\} \Lambda_{\mu, \nu}(\mathbf{z} ; \eta)
$$

provided that each member of (4.3) exists.

Proof. Let $T$ denote the left-hand side of the equality (4.3) of Theorem 1. Then, upon substituting the polynomials

$$
\Theta_{n, p, \mu, v}\left(\mathbf{x}, \mathbf{y} ; \mathbf{z} ; \frac{\eta}{t^{p}}\right)
$$

from definition (4.2) into the left-hand side of (4.3), we find

$$
T=\sum_{n=0}^{\infty} \sum_{k=0}^{[n / p]} a_{k} P_{n-p k}^{\left(A_{1}, \ldots, A_{r}\right)}(\mathbf{m}, \mathbf{x}, \mathbf{y}, \mathbf{C}) \Omega_{\mu+v k}(\mathbf{z}) \eta^{k} t^{n-p k} .
$$

Replacing $n$ by $n+p k$, we can write

$$
\begin{aligned}
T & =\sum_{n=0}^{\infty} \sum_{k=0}^{\infty} a_{k} P_{n}^{\left(A_{1}, \ldots, A_{r}\right)}(\mathbf{m}, \mathbf{x}, \mathbf{y}, \mathbf{C}) \Omega_{\mu+v k}(\mathbf{z}) \eta^{k} t^{n} \\
& =\sum_{n=0}^{\infty} P_{n}^{\left(A_{1}, \ldots, A_{r}\right)}(\mathbf{m}, \mathbf{x}, \mathbf{y}, \mathbf{C}) t^{n} \sum_{k=0}^{\infty} a_{k} \Omega_{\mu+v k}(\mathbf{z}) \eta^{k} \\
& =\prod_{i=1}^{r}\left\{\left(C_{i}-m_{i} x_{i} t+y_{i} t^{m_{i}}\right)^{-A_{i}}\right\} \Lambda_{\mu, v}(\mathbf{z} ; \eta),
\end{aligned}
$$

which completes the proof.

In a similar manner, we can give the next result.

Theorem 2. For a non-vanishing function $\Omega_{\mu}(\mathbf{z})$ of $s$ complex variables $z_{1}, \ldots z_{s}(s \in \mathbb{N})$ and for $p \in \mathbb{N}, \mu, v \in \mathbb{C}, \mathbf{z}=\left(z_{1}, \ldots, z_{s}\right), A:=\left(A_{1}, \ldots, A_{r}\right)$, $B:=\left(B_{1}, \ldots, B_{r}\right), A_{i}, B_{i} \in \mathbb{C}^{N \times N}$ for $i=1,2, \ldots, r$, let

$$
\Xi_{\mu, \nu, \mathbf{C}, \mathbf{m}}^{n, p}(\mathbf{x}, \mathbf{y} ; \mathbf{z} ; w):=\sum_{k=0}^{[n / p]} a_{k} P_{n-p k}^{\left(A_{1}+B_{1}, \ldots, A_{r}+B_{r}\right)}(\mathbf{m}, \mathbf{x}, \mathbf{y}, \mathbf{C}) \Omega_{\mu+\nu k}(\mathbf{z}) w^{k}
$$

where $a_{k} \neq 0 ; n, k \in \mathbb{N}_{0} ; \mathbb{N}_{0}:=\mathbb{N} \cup\{0\}$. Then we have

$$
\begin{aligned}
& \sum_{k=0}^{n} \sum_{l=0}^{[k / p]} a_{l} P_{n-k}^{\left(A_{1}, \ldots, A_{r}\right)}(\mathbf{m}, \mathbf{x}, \mathbf{y}, \mathbf{C}) P_{k-p l}^{\left(B_{1}, \ldots, B_{r}\right)}(\mathbf{m}, \mathbf{x}, \mathbf{y}, \mathbf{C}) \Omega_{\mu+v l}(\mathbf{z}) w^{l} \\
= & \Xi_{\mu, v, \mathbf{C}, \mathbf{m}}^{n, p}(\mathbf{x}, \mathbf{y} ; \mathbf{z} ; w)
\end{aligned}
$$


provided that each member of (4.6) exists where the matrices commute with each other.

\section{SPECIAL CASES AND SOME FURTHER PROPERTIES}

It is possible to give many applications of the theorems obtained in the previous sections with the help of appropriate choices of the multivariable functions $\Omega_{\mu+v k}(\mathbf{z})$, $\mathbf{z}=\left(z_{1}, \ldots, z_{s}\right), k \in \mathbb{N}_{0}, s \in \mathbb{N}$. For example, if we set

$$
s=r \text { and } \Omega_{\mu+\nu k}(\mathbf{z})=h_{\mu+\nu k}^{\left(B_{1}, \ldots, B_{r}\right)}(\mathbf{z})
$$

in Theorem 1, where the matrix version of the multivariable Lagrange-Hermite polynomials

$$
h_{n}^{\left(B_{1}, \ldots, B_{r}\right)}(\mathbf{x})
$$

are generated by (2.5), then we obtain the following result which provides a class of bilateral generating matrix functions for the matrix version of the multivariable Lagrange-Hermite polynomials and for the matrix version of the multivariable Humbert polynomials given explicitly by (2.2).

Corollary 1. If $\Lambda_{\mu, v}(\mathbf{z} ; w):=\sum_{k=0}^{\infty} a_{k} h_{\mu+v k}^{\left(B_{1}, \ldots, B_{r}\right)}(\mathbf{z}) w^{k}, a_{k} \neq 0, \mu, v \in \mathbb{N}_{0}$, $\mathbf{z}=\left(z_{1}, \ldots, z_{r}\right)$ and

$$
\Theta_{n, p, \mu, v}(\mathbf{x}, \mathbf{y} ; \mathbf{z} ; \zeta):=\sum_{k=0}^{[n / p]} a_{k} P_{n-p k}^{\left(A_{1}, \ldots, A_{r}\right)}(\mathbf{m}, \mathbf{x}, \mathbf{y}, \mathbf{C}) h_{\mu+v k}^{\left(B_{1}, \ldots, B_{r}\right)}(\mathbf{z}) \zeta^{k}
$$

where $n \in \mathbb{N}_{0} ; p \in \mathbb{N} ; A_{i}, B_{i} \in \mathbb{C}^{N \times N} ; \mathbf{x}=\left(x_{1}, \ldots, x_{r}\right) ; \mathbf{y}=\left(y_{1}, \ldots, y_{r}\right)$; $\mathbf{C}=\left(C_{1}, \ldots, C_{r}\right) ; \mathbf{m}=\left(m_{1}, \ldots, m_{r}\right), m_{i}=1,2, \ldots(i=1,2, \ldots, r)$, then

$$
\sum_{n=0}^{\infty} \Theta_{n, p, \mu, \nu}\left(\mathbf{x}, \mathbf{y} ; \mathbf{z} ; \frac{\eta}{t^{p}}\right) t^{n}=\prod_{i=1}^{r}\left\{\left(C_{i}-m_{i} x_{i} t+y_{i} t^{m_{i}}\right)^{-A_{i}}\right\} \Lambda_{\mu, \nu}(\mathbf{z} ; \eta)
$$

provided that each member of (5.1) exists.

Remark 1. Using the generating relation (2.5) for the matrix version of the multivariable Lagrange-Hermite polynomials and setting $a_{k}=1, \mu=0, v=1$, we obtain

$$
\begin{aligned}
& \sum_{n=0}^{\infty} \sum_{k=0}^{[n / p]} P_{n-p k}^{\left(A_{1}, \ldots, A_{r}\right)}(\mathbf{m}, \mathbf{x}, \mathbf{y}, \mathbf{C}) h_{k}^{\left(B_{1}, \ldots, B_{r}\right)}(\mathbf{z}) \eta^{k} t^{n-p k} \\
= & \left(\prod_{i=1}^{r}\left(C_{i}-m_{i} x_{i} t+y_{i} t^{m_{i}}\right)^{-A_{i}}\right)\left(\prod_{i=1}^{r}\left(1-z_{i} \eta^{i}\right)^{-B_{i}}\right),
\end{aligned}
$$

where

$$
|\eta|<\min \left\{\left|z_{1}\right|^{-1},\left|z_{2}\right|^{-1 / 2}, \ldots,\left|z_{r}\right|^{-1 / r}\right\},
$$




$$
\left|m_{i} x_{i} t-y_{i} t^{m_{i}}\right|<\left|C_{i}\right| ; i=1,2, \ldots, r .
$$

Also, if we choose $s=2 r$ and $\Omega_{\mu+\nu k}(\mathbf{z})=P_{\mu+\nu k}^{\left(E_{1}, \ldots, E_{r}\right)}(\mathbf{m}, \mathbf{t}, \omega, \mathbf{C}), \mu, v \in \mathbb{N}_{0}, \mathbf{t}=$ $\left(t_{1}, \ldots, t_{r}\right), \omega=\left(\omega_{1}, \ldots, \omega_{r}\right)$ in Theorem 2 , we obtain the following class of bilinear generating matrix functions for the matrix version of the multivariable Humbert polynomials given explicitly by (2.2).

Corollary 2. If

$$
\begin{aligned}
& \Lambda_{\mu, \nu, \mathbf{C}, \mathbf{m}}^{n, p}(\mathbf{x}, \mathbf{y} ; \mathbf{t}, \omega ; w) \\
:= & \sum_{k=0}^{[n / p]} a_{k} P_{n-p k}^{\left(A_{1}+B_{1}, \ldots, A_{r}+B_{r}\right)}(\mathbf{m}, \mathbf{x}, \mathbf{y}, \mathbf{C}) P_{\mu+\nu k}^{\left(E_{1}, \ldots, E_{r}\right)}(\mathbf{m}, \mathbf{t}, \omega, \mathbf{C}) w^{k} \\
\left(a_{k} \neq\right. & \left.0 ; p \in \mathbb{N} ; n, k, \mu, \nu \in \mathbb{N}_{0}\right)
\end{aligned}
$$

where $A_{i}, B_{i}, E_{i} \in \mathbb{C}^{N \times N}$ for $i=1,2, \ldots, r$, then

$$
\begin{aligned}
& \sum_{k=0}^{n} \sum_{l=0}^{[k / p]} a_{l} P_{n-k}^{\left(A_{1}, \ldots, A_{r}\right)}(\mathbf{m}, \mathbf{x}, \mathbf{y}, \mathbf{C}) \\
& P_{k-p l}^{\left(B_{1}, \ldots, B_{r}\right)}(\mathbf{m}, \mathbf{x}, \mathbf{y}, \mathbf{C}) P_{\mu+\nu l}^{\left(E_{1}, \ldots, E_{r}\right)}(\mathbf{m}, \mathbf{t}, \omega, \mathbf{C}) w^{l} \\
= & \Lambda_{\mu, \nu, \mathbf{C}, \mathbf{m}}^{n, p}(\mathbf{x}, \mathbf{y} ; \mathbf{t}, \omega ; w)
\end{aligned}
$$

provided that each member of (5.2) exists where $A_{i} B_{j}=B_{j} A_{i}$ for $i, j=1,2, \ldots, r$.

For example, if we set

$$
s=1 \text { and } \Omega_{\mu+v k}(y)=L_{\mu+\nu k}^{(E, \lambda)}(y)
$$

in Theorem 1, where the $n$th Laguerre matrix polynomials $L_{n}^{(E, \lambda)}(x)$ are defined by [18]

$$
L_{n}^{(E, \lambda)}(x)=\sum_{k=0}^{n} \frac{(-1)^{k} \lambda^{k}}{k !(n-k) !}(E+I)_{n}\left[(E+I)_{k}\right]^{-1} x^{k},
$$

where $E$ is a matrix in $\mathbb{C}^{N \times N}, E+n I$ is invertible for every integer $n \geq 0$ and $\lambda$ is a complex number with $\Re(\lambda)>0$ and they are generated by

$$
\begin{aligned}
\sum_{n=0}^{\infty} L_{n}^{(E, \lambda)}(x) t^{n} & =(1-t)^{-(E+I)} \exp \left(\frac{-\lambda x t}{1-t}\right), \\
|t| & <1,0<x<\infty,
\end{aligned}
$$

then we obtain the following result which provides a class of bilateral generating matrix functions for the matrix version of the multivariable Humbert and Laguerre matrix polynomials. 
Corollary 3. If $\Lambda_{\mu, v}(z ; w):=\sum_{k=0}^{\infty} a_{k} L_{\mu+\nu k}^{(E, \lambda)}(z) w^{k}$ where $\left(a_{k} \neq 0, \mu, v \in \mathbb{N}_{0}\right)$; and

$$
\Theta_{n, p, \mu, \nu}(\mathbf{x}, \mathbf{y} ; z ; \zeta):=\sum_{k=0}^{[n / p]} a_{k} P_{n-p k}^{\left(A_{1}, \ldots, A_{r}\right)}(\mathbf{m}, \mathbf{x}, \mathbf{y}, \mathbf{C}) L_{\mu+\nu k}^{(E, \lambda)}(z) \zeta^{k}
$$

where $n, p \in \mathbb{N}$. Then we have

$$
\sum_{n=0}^{\infty} \Theta_{n, p, \mu, \nu}\left(\mathbf{x} ; \mathbf{y} ; z ; \frac{\eta}{t^{p}}\right) t^{n}=\prod_{i=1}^{r}\left\{\left(C_{i}-m_{i} x_{i} t+y_{i} t^{m_{i}}\right)^{-A_{i}}\right\} \Lambda_{\mu, \nu}(z ; \eta)
$$

provided that each member of (5.4) exists.

Remark 2. Using the generating relation (5.3) for the Laguerre matrix polynomials and taking $a_{k}=1, \mu=0, v=1$, we have

$$
\begin{aligned}
& \sum_{n=0}^{\infty} \sum_{k=0}^{[n / p]} P_{n-p k}^{\left(A_{1}, \ldots, A_{r}\right)}(\mathbf{m}, \mathbf{x}, \mathbf{y}, \mathbf{C}) L_{k}^{(E, \lambda)}(z) \eta^{k} t^{n-p k} \\
= & \prod_{i=1}^{r}\left\{\left(C_{i}-m_{i} x_{i} t+y_{i} t^{m_{i}}\right)^{-A_{i}}\right\} \\
& \times(1-\eta)^{-(E+I)} \exp \left(\frac{-\lambda z \eta}{1-\eta}\right),
\end{aligned}
$$

where $|\eta|<1, \quad 0<z<\infty$.

Remark 3. For $r=1$ in (5.5), we have a bilateral generating matrix function of the Humbert (2.3) and Laguerre matrix polynomials:

$$
\begin{aligned}
& \sum_{n=0}^{\infty} \sum_{k=0}^{[n / p]} P_{n-p k}^{(A)}(m, x, y, C) L_{k}^{(E, \lambda)}(z) \eta^{k} t^{n-p k} \\
= & \left(C-m x t+y t^{m}\right)^{-A}(1-\eta)^{-(E+I)} \exp \left(\frac{-\lambda z \eta}{1-\eta}\right) .
\end{aligned}
$$

Remark 4. For $r=1$ and $s=2$ in Theorem 1, setting $\Omega_{\mu+v k}(z)=P_{n}^{(B)}(m, x, y, C)\left(B \in \mathbb{C}^{N \times N}\right)$ and taking $a_{k}=1, \mu=0, v=1$, we have bilinear generating matrix function for the Humbert matrix polynomials:

$$
\begin{aligned}
& \sum_{n=0}^{\infty} \sum_{k=0}^{[n / p]} P_{n-p k}^{(A)}(m, x, y, C) P_{k}^{(B)}(m, x, y, C) \eta^{k} t^{n-p k} \\
= & \left(C-m x t+y t^{m}\right)^{-A}\left(C-m x \eta+y \eta^{m}\right)^{-B} .
\end{aligned}
$$


Furthermore, for every suitable choice of the coefficients $a_{k}\left(k \in \mathbb{N}_{0}\right)$, if the multivariable function $\Omega_{\mu+v k}(\mathbf{y}), \mathbf{y}=\left(y_{1}, \ldots, y_{s}\right),(s \in \mathbb{N})$, is expressed as an appropriate product of several simpler functions, then the assertions of Theorems 1 and 2 can be applied in order to derive various families of multilinear and multilateral generating matrix functions for the matrix version of the multivariable Humbert polynomials given explicitly by (2.2).

We now discuss some further properties of matrix version of the multivariable Humbert polynomials given by (2.2). First of all, the generating matrix relation (2.1) yields the following addition formula for these multivariable polynomials:

$$
\begin{gathered}
P_{n}^{\left(A_{1}+B_{1}, \ldots, A_{r}+B_{r}\right)}(\mathbf{m}, \mathbf{x}, \mathbf{y}, \mathbf{C}) \\
=\sum_{k=0}^{n} P_{n-k}^{\left(A_{1}, \ldots, A_{r}\right)}(\mathbf{m}, \mathbf{x}, \mathbf{y}, \mathbf{C}) P_{k}^{\left(B_{1}, \ldots, B_{r}\right)}(\mathbf{m}, \mathbf{x}, \mathbf{y}, \mathbf{C})
\end{gathered}
$$

where $A_{i}, B_{i} \in \mathbb{C}^{N \times N}, A_{i} B_{j}=B_{j} A_{i}$ for $i, j=1,2, \ldots, r$.

On the other hand, the multivariable Humbert matrix polynomials satisfy the following equation:

$$
\sum_{j=1}^{r}\left(x_{j} \frac{\partial}{\partial x_{j}}+m_{j} y_{j} \frac{\partial}{\partial y_{j}}\right) P_{n}^{\left(A_{1}, \ldots, A_{r}\right)}(\mathbf{m}, \mathbf{x}, \mathbf{y}, \mathbf{C})=n P_{n}^{\left(A_{1}, \ldots, A_{r}\right)}(\mathbf{m}, \mathbf{x}, \mathbf{y}, \mathbf{C}) .
$$

If we differentiate each member of the generating function (2.1) with respect to $x_{j}$ and $y_{j}(j=1,2, \ldots, r)$, we obtain the following (differential) recurrence relations for the matrix version of the multivariable Humbert polynomials:

$$
\begin{aligned}
& \frac{\partial}{\partial x_{j}} P_{n}^{\left(A_{1}, \ldots, A_{r}\right)}(\mathbf{m}, \mathbf{x}, \mathbf{y}, \mathbf{C}) \\
= & \sum_{k=0}^{n-1} \sum_{l=0}^{\left[k / m_{j}\right]} \frac{(-1)^{l}\left(k+l-l m_{j}\right) ! A_{j}\left(m_{j}\right)^{k-l m_{j}+1}}{\left(k-l m_{j}\right) ! l ! C_{j}^{k-l\left(m_{j}-1\right)+1}} x_{j}^{k-l m_{j}} y_{j}^{l} \\
& \times P_{n-k-1}^{\left(A_{1}, \ldots, A_{r}\right)}(\mathbf{m}, \mathbf{x}, \mathbf{y}, \mathbf{C})
\end{aligned}
$$

for $n \geq 1$, and

$$
\begin{aligned}
& \frac{\partial}{\partial y_{j}} P_{n}^{\left(A_{1}, \ldots, A_{r}\right)}(\mathbf{m}, \mathbf{x}, \mathbf{y}, \mathbf{C}) \\
= & -\sum_{k=0}^{n-m_{j}} \sum_{l=0}^{\left[k / m_{j}\right]} \frac{(-1)^{l}\left(k+l-l m_{j}\right) ! A_{j}\left(m_{j}\right)^{k-l m_{j}}}{\left(k-l m_{j}\right) ! l ! C_{j}^{k-l\left(m_{j}-1\right)+1}} x_{j}^{k-l m_{j}} y_{j}^{l} \\
& \times P_{n-k-m_{j}}^{\left(A_{1}, \ldots, A_{r}\right)}(\mathbf{m}, \mathbf{x}, \mathbf{y}, \mathbf{C})
\end{aligned}
$$


where $n \geq m_{j}$ and $m_{j}(j=1,2, \ldots, r)$ is a positive integer and all matrices are commutative. By applying (5.6), (5.7) and (5.8), the following recurrence relation for the matrix polynomials (given explicitly by (2.2)) can be easily derived:

$$
\begin{aligned}
& \sum_{j=1}^{r} \sum_{k=0}^{n-1} \sum_{l=0}^{\left[k / m_{j}\right]} \frac{(-1)^{l}\left(k+l-l m_{j}\right) ! A_{j}\left(m_{j}\right)^{k-l m_{j}+1}}{\left(k-l m_{j}\right) l ! C_{j}^{k-l\left(m_{j}-1\right)+1}} \\
& x_{j}^{k-l m_{j}+1} y_{j}^{l} P_{n-k-1}^{\left(A_{1}, \ldots, A_{r}\right)}(\mathbf{m}, \mathbf{x}, \mathbf{y}, \mathbf{C}) \\
& -\sum_{j=1}^{r} \sum_{k=0}^{n-m_{j}} \sum_{l=0}^{\left[k / m_{j}\right]} \frac{(-1)^{l}\left(k+l-l m_{j}\right) ! A_{j}\left(m_{j}\right)^{k-l m_{j}+1}}{\left(k-l m_{j}\right) ! l ! C_{j}^{k-l\left(m_{j}-1\right)+1}} \\
& x_{j}^{k-l m_{j}} y_{j}^{l+1} P_{n-k-m_{j}}^{\left(A_{1}, \ldots, A_{r}\right)}(\mathbf{m}, \mathbf{x}, \mathbf{y}, \mathbf{C}) \\
= & n P_{n}^{\left(A_{1}, \ldots, A_{r}\right)}(\mathbf{m}, \mathbf{x}, \mathbf{y}, \mathbf{C}), \text { for } n \geq m_{j}
\end{aligned}
$$

where $m_{j}(j=1,2, \ldots, r)$ is a positive integer and all matrices commute.

\section{ACKNOWLEDGEMENT}

The authors are grateful to the referee(s) for their valuable comments and suggestions which improved the quality and the clarity of the paper.

\section{REFERENCES}

[1] R. Aktaş, R. Şahin, and A. Altın, "On a multivariable extension of the Humbert polynomials," Appl. Math. Comput., vol. 218, no. 3, pp. 662-666, 2011.

[2] A. Altin and E. Erkuş, "On a multivariable extension of the Lagrange-Hermite polynomials," Integral Transforms Spec. Funct., vol. 17, no. 4, pp. 239-244, 2006.

[3] W. C. C. Chan, C. J. Chyan, and H. M. Srivastava, "The Lagrange polynomials in several variables," Integral Transforms Spec. Funct., vol. 12, no. 2, pp. 139-148, 2001.

[4] G. Dattoli, P. E. Ricci, and C. Cesarano, "The Lagrange polynomials, the associated generalizations, and the umbral calculus," Integral Transforms Spec. Funct., vol. 14, no. 2, pp. 181-186, 2003.

[5] E. Defez, A. Hervás, A. Law, J. Villanueva-Oller, and R. J. Villanueva, "Progressive transmission of images: PC-based computations, using orthogonal matrix polynomials," Math. Comput. Modelling, vol. 32, no. 10, pp. 1125-1140, 2000.

[6] E. Defez, L. Jódar, and A. Law, "Jacobi matrix differential equation, polynomial solutions, and their properties," Comput. Math. Appl., vol. 48, no. 5-6, pp. 789-803, 2004.

[7] E. Defez, L. Jódar, A. Law, and E. Ponsoda, "Three-term recurrences and matrix orthogonal polynomials," Util. Math., vol. 57, pp. 129-146, 2000.

[8] E. Defez, J. Villanueva-Oller, R. Villanueva, and A. Law, "Matrix cubic splines for progressive 3D imaging," J. Math. Imaging Vis., vol. 17, no. 1, pp. 41-53, 2002.

[9] E. Defez and L. Jódar, "Some applications of the Hermite matrix polynomials series expansions," J. Comput. Appl. Math., vol. 99, no. 1-2, pp. 105-117, 1998.

[10] E. Defez and L. Jódar, "Chebyshev matrix polynomials and second order matrix differential equations," Util. Math., vol. 61, pp. 107-123, 2002. 
[11] A. J. Duran, "On orthogonal polynomials with respect to a positive definite matrix of measures," Can. J. Math., vol. 47, no. 1, pp. 88-112, 1995.

[12] A. J. Duran and P. Lopez-Rodriguez, "Orthogonal matrix polynomials: zeros and Blumenthal's theorem," J. Approximation Theory, vol. 84, no. 1, pp. 96-118, 1996.

[13] E. Erkuş and H. M. Srivastava, "A unified presentation of some families of multivariable polynomials," Integral Transforms Spec. Funct., vol. 17, no. 4, pp. 267-273, 2006.

[14] E. Erkuş-Duman, "Matrix extensions of polynomials in several variables," Util. Math., vol. 85, pp. 161-180, 2011.

[15] J. S. Geronimo, "Scattering theory and matrix orthogonal polynomials on the real line," Circuits Syst. Signal Process, vol. 1, pp. 471-495, 1982.

[16] H. W. Gould, "Inverse series relations and other expansions involving Humbert polynomials," Duke Math. J., vol. 32, pp. 697-711, 1965.

[17] A. T. James, "Special functions of matrix and single argument in statistics," Theory Appl. spec. Funct., Proc. adv. Semin., Madison, pp. 497-520, 1975.

[18] L. Jódar, R. Company, and E. Navarro, "Laguerre matrix polynomials and systems of second-order differential equations," Appl. Numer. Math., vol. 15, no. 1, pp. 53-63, 1994.

[19] L. Jódar, R. Company, and E. Ponsoda, "Orthogonal matrix polynomials and systems of second order differential equations," Differ. Equ. Dyn. Syst., vol. 3, no. 3, pp. 269-288, 1995.

[20] L. Jódar and J. C. Cortés, "On the hypergeometric matrix function," J. Comput. Appl. Math., vol. 99, no. 1-2, pp. 205-217, 1998.

[21] L. Jódar and E. Defez, "A connection between Laguerre's and Hermite's matrix polynomials," Appl. Math. Lett., vol. 11, no. 1, pp. 13-17, 1998.

[22] L. Jódar, E. Defez, and E. Ponsoda, "Matrix quadrature integration and orthogonal matrix polynomials," Congr. Numerantium, vol. 106, pp. 141-153, 1995.

[23] A. Sinap and W. Van Assche, "Polynomial interpolation and Gaussian quadrature for matrixvalued functions," Linear Algebra Appl., vol. 207, pp. 71-114, 1994.

[24] R. C. Singh Chandel and H. C. Yadava, "A binomial analogue of Srivastava's theorem," Indian J. Pure Appl. Math., vol. 15, pp. 383-386, 1984.

[25] H. M. Srivastava, "Some generalizations of Carlitz's theorem," Pac. J. Math., vol. 85, pp. 471-477, 1979.

[26] H. M. Srivastava and H. L. Manocha, A treatise on generating functions, ser. Ellis Horwood Series in Mathematics and Its Applications. Chichester: Ellis Horwood Limited; New York: Halsted Press: a Division of John Wiley \& Son, 1984.

Authors' addresses

\section{Rabia Aktaş}

Ankara University, Faculty of Science, Department of Mathematics, Tandoğan TR-06100, Ankara, Turkey

E-mail address: raktasescience.ankara.edu.tr

\section{Bayram Çekim}

Gazi University, Faculty of Science, Department of Mathematics, Teknik Okullar TR-06500, Ankara, Turkey

E-mail address: bayramcekimegazi.edu.tr

\section{Recep Şahin}

Ankara University, Faculty of Science, Department of Mathematics, Tandoğan TR-06100, Ankara, Turkey

E-mail address: sahin@science.ankara.edu.tr 